\title{
Bisphosphonates and metastasis
}

\author{
J. Lester, R. Coleman \\ Academic Unit of Clinical Oncology, Weston Park Hospital, Sheffield, UK.
}

Abstract Bone metastases frequently occur in advanced breast cancer patients and once incorporated into bone have profound effects on bone physiology. Bisphosphonates are potent inhibitors of osteoclastic function and are becoming increasingly important for the management of patients with breast cancer. They affect the ability of cancer cells to accelerate bone turnover and as a result control pain and reduce the risk of pathological fracture.

Furthermore ongoing clinical trials are investigating the role of bisphosphonates as an adjuvant therapy in high-risk breast cancer patients with an aim to prevent bone metastasis.

This article reviews the mechanisms of bone metastasis and will discuss present and future developments for the monitoring and treatment of metastatic bone disease.

Keywords: Bisphosphonates; Breast cancer; Bone metastasis; Adjuvant therapy

\section{Introduction}

In recent years the increasing use of adjuvant chemotherapy and endocrine therapy have resulted in a decline in breast cancer mortality despite a steady increase in incidence [1].

Bone is a common site for the distant spread of breast cancer with as many as $70 \%$ of breast cancer patients having bone metastasis at post-mortem. Significant morbidity from bone metastasis may occur due to pathological fracture, hypercalcaemia of malignancy, pain and spinal cord compression [2].

The bisphosphonates are potent inhibitors of osteoclast function. There are two different classes of bisphosphonates; the nitrogen containing and the non-nitrogen containing compounds. Nitrogen containing bisphosphonates include pamidronate, alendronate, risedronate, zoledronic acid and ibandronate. These drugs have the greatest affinity and potency and act through the inhibition of farnesyl diphosphate

Correspondence to: Prof. Robert Coleman, MD, FRCP, Academic Unit of Clinical Oncology, Cancer Research Centre, Weston Park Hospital, Sheffield S10 2SJ, UK. E-mail: R.E.Coleman@sheffield.ac.uk

Publication date 28/01/05 BCO/267/2004/FO
(FPP) synthase in the mevalonate pathway. Nonnitrogen containing bisphosphonates include etidronate and clodronate, with the latter working through effects on mitochondrial ATP in osteoclasts.

The exact mechanisms of action remain unclear. However, animal studies with a variety of tumour models suggest that bisphosphonates may stimulate apoptosis of both the tumour cell and the osteoclast [3-5].

\section{Bone turnover}

Bone is constantly undergoing a process of renewal known as remodelling. This process of bone resorption followed by formation is a delicate balance between two cell types, the osteoclast and the osteoblast. Control of these cell types is regulated by a combination of systemic hormones and local factors that affect the replication and differentiation of osteoblasts and osteoclasts.

Growth factors, such as insulin-like growth factors (IGF-I and IGF-II), transforming growth factor- $\beta$ (TGF- $\beta$ ), fibroblast growth factors (FGFs) and plateletderived growth factor (PDGF) mainly act to increase bone formation and repair. Cytokines involved in 
bone remodelling include interleukin (IL) -1, -4, -6 and -11 , macrophage colony-stimulating factor (MCSF), granulocyte-macrophage colony-stimulating factor (GMCSF) and tumour necrosis factor (TNF). These cytokines all stimulate the recruitment of osteoclasts and therefore the resorption of bone.

Over the last few years it has become clear that the major regulation of osteoclast activity is via TNF ligand, receptor activator of NF-kappa B (RANK) ligand (RANKL) and its two known receptors RANK and osteoprotegerin (OPG). Cytokines all stimulate transcription of RANKL, which in combination with RANK facilitates osteoclastic activity. Conversely OPG acts as a decoy receptor to RANKL and halts its activity $[6,7]$.

\section{Development of bone metastasis}

Once a breast cancer cell incorporates into bone it interferes with normal bone homoeostasis by the secretion of a variety of growth factors and cytokines that include parathyroid hormone-related protein (PTHrP). PTHrP enhances osteoclastic activity and therefore bone resorption [8]. Growth factors, such as TGF- $\beta$ and IGF upregulate RANKL, and in turn indirectly stimulate osteoclastogenesis $[9,10]$. This theory is strengthened by animal studies, which show that the bone destruction occurring within osteolytic bone metastasis is mediated by increased osteoclastic activity rather than direct tumour cell invasion [11].

Actively resorbing bone releases a number of factors (such as TGF- $\beta$ ) that can both attract cancer cells to the bone surface and facilitate their growth and proliferation [12]. This creates a vicious circle whereby a tumour cell is able to stimulate the bone micro-environment which in turn stimulates the development of the tumour (See Fig. 1). The inhibition of bone resorption should therefore have an effect on the development and progression of metastatic disease.

\section{Bone turnover markers}

As discussed in the previous section, tumour cells increase the rate of bone resorption by the stimulation of osteoclasts. This activity in turn stimulates bone formation and turnover. When bone is formed or broken down various markers are released into the circulation that correlate with the rate of bone resorption and formation. Bisphosphonates reduce osteoclastic activity and as a result lower bone resorption and formation markers, which can then be used as a guide for treatment.

Type I collagen, the major component of bone is broken down into many different substrates that can be measured as highly specific markers of bone resorption. These include the $\mathrm{N}$-terminal (NTX) and C-terminal (CTX) peptide-bound crosslinks of type I collagen and can be measured in both the serum and urine.

Studies have shown that high levels of NTX correlate with a shorter duration of time to progression, skeletal-related events (SREs) and survival $[13,14]$. Serial measurements of bone turnover markers can be used as a guide to the frequency and dosage of bisphosphonate drug used. A study by Costa et al. [15] evaluated the role of bone turnover markers for the monitoring of metastatic breast cancer patients. Of the markers studied, urinary NTX (UNTX) showed the most diagnostic accuracy and was able to predict the progression of metastatic disease most reliably.

Studies using pamidronate have shown that a reduction in UNTX correlates with good pain control [16] and a trend towards a reduction in fractures $(P=0.07)$ [17]. As in the treatment of benign bone diseases, such as osteoporosis, the aim of treatment should be to maintain low levels of NTX.

\section{Bisphosphonates for the treatment of bone metastasis}

A variety of treatments are available for patients with metastatic bone disease. These include chemotherapy, radiotherapy, hormonal therapy, orthopaedic surgery and bisphosphonates. Patients with bone metastasis alone have a median survival of 2 years [2] but are at risk of SREs that cause significant morbidity and mortality during this period. The addition of bisphosphonates to other breast cancer therapies can reduce the risk of these events but questions still remain regarding when to treat and for how long.

All bisphosphonates have been shown to reduce the frequency of SREs from metastatic bone disease, and with many of them important effects on symptoms have been reported with reduced pain and analgesic consumption and an apparent improvement in quality of life [18-20]. Third generation bisphosphonates, such as ibandronate and zoledronic acid are more potent than pamidronate and are more convenient due to shorter infusion times.

A large phase III study has compared the efficacy of intravenous (i.v.) zoledronic acid and pamidronate in patients with metastatic breast cancer. In this trial 1130 patients were treated with either $4 \mathrm{mg}$ zoledronate, $8 \mathrm{mg}$ zoledronate or $90 \mathrm{mg}$ pamidronate. Multiple event analysis was used to capture data on all clinically relevant SREs and the time to each event. Zoledronic acid reduced the risk of skeletal complications by $20 \%(P=0.037)$. Total numbers of SREs and time to first event were similar in both 


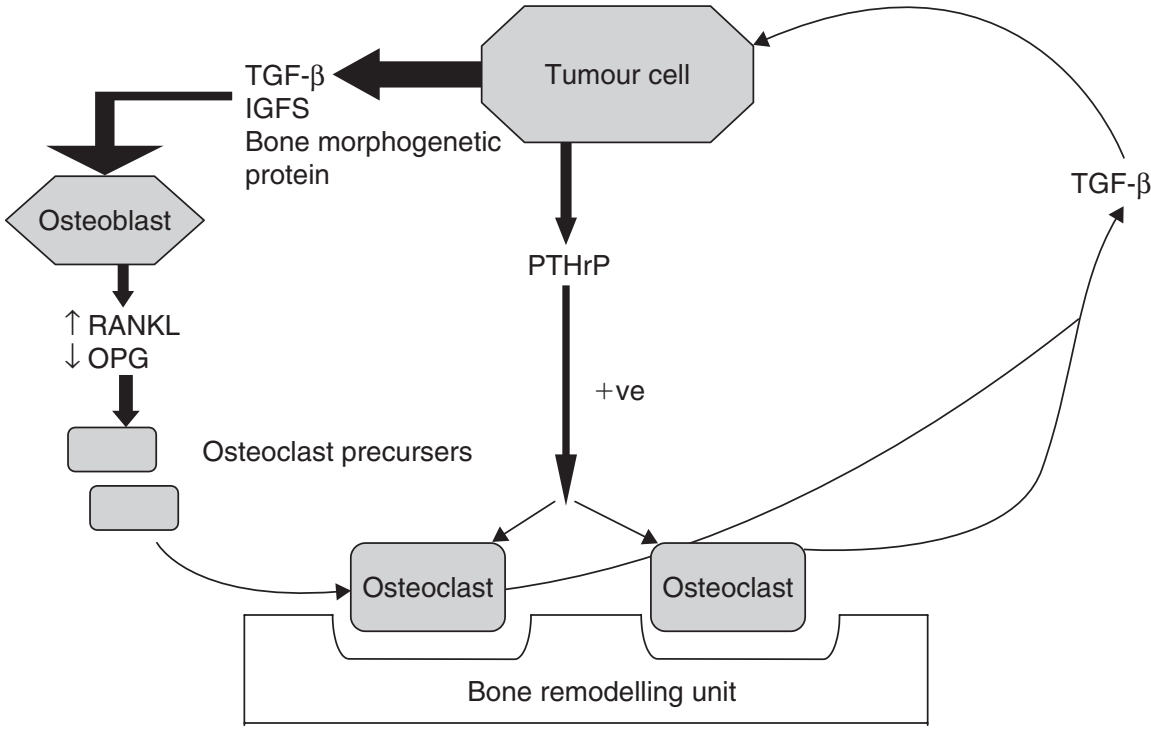

Figure 1.

Bone remodelling unit. groups. However, within a subgroup of patients with osteolytic metastasis, zoledronic acid resulted in a significant improvement in the time to first SRE $(P=0.013)$. Zoledronic acid also resulted in even greater reductions in UNTX levels compared to pamidronate $(P=0.015)[21,22]$.

Ibandronate, another third generation bisphosphonate, is also emerging as a useful drug for the treatment of metastatic breast cancer. Trials comparing i.v. ibandronate to placebo have recently been published. The skeletal morbidity period rate (SMPR) which evaluates the number of new skeletal complications within a 12-week period, and is a composite endpoint comprising vertebral and non-vertebral fractures, and the need for radiotherapy or surgery was used to assess treatment response. The $6 \mathrm{mg}$ ibandronate dose was associated with a $20 \%$ reduction in the SMPR compared to placebo. Significant decreases in new bone events and increases in time to first event were also apparent [23].

Ibandronate can also be administered orally and has also been compared to placebo. Significant advantages were again observed for SMPR in treated patients $(P=0.004)$ with a $38 \%$ risk reduction for skeletal events $(P<0.0001)$. Urinary CTX (uCTX) levels declined significantly in patients treated with ibandronate compared to placebo $(P<0.0001)$. Despite these promising results there was no significant difference in the number of skeletal fractures $(P=0.195)$ and only a trend towards a delay in the time to the first SRE (90.3 vs. 64.0 weeks; $P=0.089$ ) [24]. A potent oral bisphosphonate is obviously an attractive prospect and studies are presently underway comparing oral ibandronate to i.v. zoledronic acid to define their respective roles in metastatic bone disease more precisely.

\section{Bisphosphonates for the adjuvant therapy of breast cancer}

Bisphosphonates have been shown to inhibit the development of bone metastasis and cause a reduction in tumour burden in animal models. It is also possible that they may prevent the future development of bone metastasis in breast cancer patients. Several studies with oral clodronate have addressed this question (See Table 1).

In the largest study by Powles et al. [25] over 1000 patients with operable breast cancer were randomized between clodronate $1600 \mathrm{mg} /$ day and placebo. Patients were treated with clodronate for 2 years, and the follow up was for 6 years in total. During the treatment phase of the study (2 years), fewer women receiving clodronate developed bone metastases compared to placebo $(n=12,2.3 \%$ vs. $n=28$, $5.2 \% ; P=0.016)$. After the treatment phase however, the difference was no longer significant $(P=0.127)$. There was no significant difference in the occurrence of non-osseous metastasis, however significant reductions in mortality were seen in patients randomized to clodronate ( $n=98$ vs. $n=129$; $P=0.047$ ).

In a study by Diel et al. [26] 302 patients with early breast cancer were recruited. Additionally these women had immunohistochemical evidence of breast cancer cells in their bone marrow and thus at high risk of developing recurrent disease. Patients were then randomized to receive clodronate $1600 \mathrm{mg} /$ day for 2 years or to standard follow-up. Following a median observation period of 36 months, the mean number of bone metastasis per patient was twice as common in the control group (6.3) compared to the treated group (3.1; $P=0.004)$. Significantly, the incidence of bone 
Table 1. Data from the adjuvant clodronate trials.

\begin{tabular}{lllllll}
\hline & Study & & & & Update at ASCO \\
Trial & number & Subjects & Design & Effect of clodronate & Significance \\
2004 [27,29]
\end{tabular}

NS: no significant difference.

and visceral metastasis were lower in patients taking clodronate, the disease-free survival was prolonged and the mortality reduced. An updated analysis of this study has recently been presented after an average follow-up of 103 months [27]. With longer follow-up, the incidence of osseous and visceral metastasis was similar in both groups. There was no longer any significant difference in disease-free survival but the improvement in overall survival was maintained in patients treated with clodronate $(P<0.01)$.

However a study by Saarto et al. [28] produced conflicting results. Two hundred and ninety-nine lymph node positive breast cancer patients were randomized to treatment with clodronate $1600 \mathrm{mg} /$ day for 3 years or to a control group. Bone metastasis was more common in the clodronate group although the difference did not reach significance (29 vs. 24 patients, respectively; $P=0.27$ ). Clodronate was also associated with an increased risk of developing nonskeletal recurrence (60 vs. 36 patients; $P=0.0007$ ). In terms of overall and disease-free survival patients treated with clodronate had worse outcomes compared to placebo $(P=0.009$ and $P=0.07$, respectively). These results were counter-intuitive and an inbalance in prognostic factors probably explains this confounding report. Ten-year follow-up results were recently presented at the ASCO 2004 conference [29]. Bone metastasis was detected at the same frequency in the clodronate and placebo groups $(P=0.35)$. Non-skeletal recurrences remained more common in patients treated with clodronate (69 vs. 51 patients; $P=0.005$ ). Ten-year disease-free survival was still less in the clodronate treated patients $(P=0.004)$, however no significant overall survival difference was now found between the two groups.

\section{Future directions}

Bisphosphonates clearly have a role in treating patients with bone metastasis from breast cancer. Questions still remain however, regarding which patients to treat, which drug to use and for how long. Potent nitrogen containing bisphosphonates, such as zoledronic acid are likely to continue to show advantages over the other less potent bisphosphonates.

Patients most likely to benefit from bisphosphonates are those with diffuse disease and a high tumour burden. These patients often have high uNTX levels and if so are most at risk of skeletal complications $[13,14]$. Further research is required to evaluate whether normalizing NTX levels in these patients improves the risk of skeletal events. Nevertheless the routine use of bone markers to stratify a group of metastatic patients in need of further investigation, and or treatment with bisphosphonates, is likely to be useful in clinical practice.

The jury is still out about the use of bisphosphonates as an adjuvant therapy. We shall have to await the results from ongoing trials involving greater numbers of patients and more potent bisphosphonates.

The National Surgical Adjuvant Breast Project (NSABP) has finished recruiting over 3000 early breast cancer patients in a placebo-controlled trial of oral clodronate.

The adjuvant zoledronic acid reduce recurrence (AZURE) study is presently recruiting node positive breast cancer patients in another randomized placebo-controlled trial. Treated patients will receive regular infusions of zoledronic acid along side standard therapy. These two trials are expected to report in 2008. 
There is evidence from animal models and in vitro experiments that bisphosphonates can induce tumour cell apoptosis and inhibit angiogenesis [30-32]. These promising early reports are interesting but further pre-clinical and clinical research will be needed to consolidate this data.

\section{References}

1. Breast Cancer. Incidence Rate Rises While Death Rate Falls. Office for National Statistics, 2004. http://www. statistics.gov.uk.

2. Coleman R, Rubens $\mathrm{D}$. The clinical course of bone metastasis from breast cancer. Br J Cancer 1987; 55: 61-66.

3. Yoneda T, Michigami T, Yi B. Use of bisphosphonates for the treatment of bone metastasis in experimental animal models. Cancer Treat Rev 1999; 25: 293-299.

4. Senaratne S, Pirianov G, Mansi J. Bisphosphonates induce apoptosis in human breast cancer cell lines. $\mathrm{Br} \mathrm{J}$ Cancer 2000; 82: 1459-1468.

5. Hiraga T, Williams $P$, Mundy G. The bisphosphonate ibandronate promotes apoptosis in MDA-MB-231 human breast cancer cells in bone metastasis. Cancer Res 2001; 61: 4418-4424.

6. Mundy G, Chen D, Oyajobi B. Bone Remodeling. Primer on the Metabolic Bone Diseases and Disorders of Mineral Metabolism, 5th Edition. Philadelphia: Lippincott, Williams and Wilkins, 2003: pp. 46-58.

7. Canalis E. Regulation of bone remodeling. Primer on the Metabolic Bone Diseases and Disorders of Mineral Metabolism, 3rd edition. New York: Lippincott-Raven; 1996: pp. 29-34.

8. Guise T, Yin J, Taylor S. Evidence for a causal role of parathyroid hormone-related protein in the pathogenesis of human breast cancer-mediated osteolysis. J Clin Invest 1996; 98: 1544-1549.

9. Kakonen S, Mundy G. Mechanisms of osteolytic bone metastasis in breast carcinoma. Cancer 2003; 97(Suppl 3): 834-839.

10. Grano M, Mori G, Minielli V. Breast cancer cell line MDA231 stimulates osteoclastogenesis and bone resorption in human osteoclasts. Biochem Biophys Res Commun 2000; 270: 1097-1100.

11. Taube T, Elomaa I, Blomqvist C. Histomorphometric evidence for osteoclast-mediated bone resorption in metastatic breast cancer. Bone 1994; 15: 161-166.

12. Yin J, Selander K, Chirgwin J. TGF-(beta) signaling blockade inhibits PTHrP secretion by breast cancer cells and bone metastasis development. J Clin Invest 1999; 103: 197-206.

13. Brown J, Thomson C, Ellis S, Gutcher SA, Purohit OP, Coleman RE. Bone resorption predicts for skeletal complications in metastatic bone disease. $\mathrm{Br} \mathrm{J}$ Cancer 2003; 89: 2031-2037.

14. Ali S, Demers L, Leitzel K, et al. Baseline serum NTx levels are prognostic in metastatic breast cancer patients with bone-only metastasis. Ann Oncol 2004; 15: 455-459.

15. Costa L, Demers L, Gouveia-Oliveira A, et al. Prospective evaluation of the peptide-bound collagen type I cross-links $\mathrm{N}$-telopeptide and $\mathrm{C}$-telopeptide in predicting bone metastasis status. J Clin Oncol 2002; 20(3): 850-856.

16. Vinholes J, Guo C, Purohit O. Metabolic effects of pamidronate in patients with metastatic bone disease. Br J Cancer 1996; 73(9): 1089-1095.
17. Lipton A, Demers L, Curley E. Markers of bone resorption in patients treated with pamidronate. Eur J Cancer 1998; 34(13): 2021-2026.

18. Hortobagyi G, Theriault R, Porter L. Efficacy of pamidronate in reducing skeletal complications in patients with breast cancer and lytic bone metastasis. New Engl J Med 1996; 335: 1785-1791.

19. Lipton A. Bisphosphonates and breast cancer. Cancer 1997; 80: 1668-1673.

20. Paterson A, Powles T, Kanis T. Double-blind controlled trial of oral clodronate in patients with bone metastasis from breast cancer. J Clin Oncol 1993; 11: 59-65.

21. Rosen L, Gordon D, Kaminski M. Zoledronic acid versus pamidronate in the treatment of skeletal metastasis in patients with breast cancer or osteolytic lesions in multiple myeloma. Cancer J 2001; 7: 377-387.

22. Rosen L, Gordon D, Dugan W. Zoledronic acid is superior to pamidronate for the treatment of bone metastasis in breast carcinoma patients with at least one osteolytic lesion. Cancer 2004; 100(1): 36-43.

23. Body J, Diel I, Lichinitser M. Intravenous ibandronate reduces the incidence of skeletal complications in patients with breast cancer and bone metastasis. Ann Oncol 2003; 14: 1399-1405.

24. Body J, Diel I, Lichinitser M. Oral ibandronate reduces the risk of skeletal complications in breast cancer patients with metastatic bone disease: results from two randomised, placebo controlled phase III studies. Br J Cancer 2004; 90(6): 1133-1137.

25. Powles TJ, Paterson S, Kanis J, et al. Randomized, placebo-controlled trial of clodronate in patients with primary operable breast cancer. J Clin Oncol 2002; 20(15): 3219-3224.

26. Diel I, Solomayer E, Costa S, et al. Reduction in new metastasis in breast cancer with adjuvent clodronate treatment. New Engl J Med 1998; 339(6): 357-363.

27. Jaschke A, Bastert G, Solomayer E, Costa S, Schuetz F, Diel I. Adjuvant clodronate treatment improves the overall survival of primary breast cancer patients with micrometastasis to bone marrow - a longtime follow-up. [Abstract 529]. J Clin Oncol 2004; 22(14S).

28. Saarto T, Blomqvist C, Virkkunen P. Adjuvant clodronate treatment does not reduce the frequency of skeletal metastasis in node-positive breast cancer patients: 5 -year results of a randomized controlled trial. J Clin Oncol 2001; 19(1): 10-17.

29. Saarto T, Vehmanen C, Blomqvist C, Elomaa I. Ten-year follow-up of a randomized controlled trial of adjuvant clodronate treatment in node-positive breast cancer patients. [Abstract 527]. J Clin Oncol 2004; 22(14S).

30. Jagadev S, Coleman R, Shipman C. The bisphosphonate zoledronic acid induces apoptosis of breast cancer cells: evidence for synergy with paclitaxel. $\mathrm{Br} \mathrm{J}$ Cancer 2001; 84: 1126-1134.

31. Wood J, Schnell C, Green J. Zoledronic acid (zometa), a potent inhibitor of bone resorption, inhibits proliferation and induces apoptosis in endothelial cell in vitro and is anti-angiogenic in a murine growth factor implant model. [Abstract 2620]. Proc Am Soc Clin Oncol 2000; 19: 664.

32. Wood J, Bonjean K, Ruetz S. Novel antiangiogenic effects of the bisphosphonate compound zoledronic acid. J Pharmacol Exp Therap 2002; 302(3): 1055-1061. 